\title{
A Baseline Environmental Impact Assessment (EIA) of Proposed Sit for Thermal Desorption Unit (TDU) Waste Management Project in Rivers State, Nigeria: Floristic Composition Approach
}

\author{
Nsirim L. Edwin-Wosu ${ }^{1}$, Victor N. Sunday ${ }^{2}$ \\ ${ }^{1}$ Department of Plant Science and Biotechnology, Faculty of Biological Science, College of Natural and Applied Sciences, University of Port \\ Harcourt, Choba, Port Harcourt, Rivers State, Nigeria \\ ${ }^{2}$ Department of Geography and Environmental Management, Faculty of Social Sciences, University of Port Harcourt, Choba, Port Harcourt, \\ Rivers State, Nigeria
}

\section{Email address:}

nsirim.edwin-wosu@uniport.edu.ng (N. L. Edwin-Wosu), victor.sunday@uniport.edu.ng (V. N. Sunday)

\section{To cite this article:}

Nsirim L. Edwin-Wosu, Victor N. Sunday. A Baseline Environmental Impact Assessment (EIA) of Proposed Sit for Thermal Desorption Unit (TDU) Waste Management Project in Rivers State, Nigeria: Floristic Composition Approach. American Journal of Bioscience and Bioengineering. Vol. 4, No. 2, 2016, pp. 9-25. doi: 10.11648/j.bio.20160402.11

Received: July 11, 2015; Accepted: August 31, 2015; Published: April 21, 2016

\begin{abstract}
This report describes the results of a wet and dry season ecological baseline impact assessment study based on the context of a proposed Thermal Desorption Unit (TDU) development project in Eteo Eleme, Rivers State. Thermal Desorption Unit project of the magnitude of a waste management project must always have some negative effects on the quality and quantity of the environment. Following an environment impact assessment (EIA) a series of mitigation measures must be put in place to minimize the intensity of the negative effect of the project on the environment. The paper seeks to assess through baseline data the envisaged impact of the project on the existing conditions of the biophysical environment, to appraise the possible risk to the environment and mitigation measures adopted. It uses a combination of standard procedures of integrated data sources to qualitatively and quantitatively assess the floristic profile of the project study area. The result highlights most of the biophysical impact variables that will have negative effect on the environment. However, the study site still maintains the status of abundance, richness and evenness with obvious similarity in floristic composition and forest structure to that in tropical forest elsewhere in the world. It is obvious that the Eteo vegetation system is gradually under ecological succession resulting to secondary vegetation system without proper articulation of its wealth of flora diversity, but with high flora diversity in rainy season than dry season. The useful application of phyto-sociological indices in determining the status of its vegetation complex in terms of species abundance, density, important value index, diversity and distribution pattern is being recorded in the Table for both wet and dry seasons. The paper emphasis the need for sound environmental commitments to the project and to appraise their implementation. A proper balance between the expected benefits from the project and cost implication can only be obtained through impact studies and careful monitoring.
\end{abstract}

Keywords: Species Diversity, Abundance, Importance Value Index, Density

\section{Introduction}

Baseline studies usually entail a collection of background information and data on the physical environment and socio-economic setting of a proposed site for development. As an inevitable factor in environmental management, it is done prior to impact assessment of the given area. It is an environmental evaluation bench mark which aid in the assessment of impacts and / or effect of existing facilities and / or on - going operation or proposed project on the environment. It is a hazard and effect management process that provides assurance for sustainable environmental practices at any project location. It seek to strike a balance between obtaining sufficient information to describe existing 
features, their inter-relationship and overall environmental status or quality, while obtainingdetailed data on the current status and trends of the vegetation to enable specific impacts be predicte [1].

A Vegetation baseline study is an aspect of Environmental Impact Assessment (EIA) for the proposed TDU project. This aim at determining the current state of the environment and assesses the likely impact of the project on the environment. Socio-ecological changes are always enshrined in every developmental activity [2]. A good monitoring and evaluation system will indicate any probable changes and results of activities, including eventual impacts and the extent of the desired results achieved and their sustainability [3, 4, 5].

Thermal desorption is a term applied to many different types of soil remediation technologies. It is an ex-situ means to physically separate volatile and some semi volatile contaminants from soil, sediments, sludges, and filter cakes. It is a thermally induced physicalseparation process. All of these technologies consist fundamentally of a two-step process, involving the application of heat to a contaminated material, such as soil, sediment, sludge, or filter cake, to vaporize the contaminants into a gas stream, which subsequently is treated to meet regulatory requirements prior to discharge [6, 7].Varieties of gas treatment technologies are used to collect, condense, or destroy these volatilized gases. Contaminants are vaporized from a solid matrix and are transferred into a gas stream where they can be more easily managed by treatment prior to discharge. Options used to manage or treat the contaminant-laden gas stream may consist of condensation, collection, or combustion. For the first two options, the condensed or collected contaminants usually are treated off site at some time subsequent to heat application, while in the third option, combustion, treatment occurs on site, immediately after the gases exit heating process $[8,9]$.

Thermal desorption is applicable to organic wastes and generally is not used for treating metals and other inorganics. Depending on the specific thermal desorption vendor selected, the technology heats contaminated media between 200 $1,000^{\circ} \mathrm{F}$, driving off water and volatile contaminants. Off-gases may be burned in an after burner, condensed to reduce the volume to be disposed, or captured by carbon adsorption beds. In addition to volatilizing organic contaminants contained in the waste feed, moisture is volatilized and leaves with the off-gas.As a result, the thermal desorption system also functions as a dryer. In fact, many vendors refer to the primary treatment chamber of their system as a "rotary dryer," highlighting its effect on the material, despite it principal purpose of evaporating and separating out the contaminants $[8,10]$.

The proposed TDU EIA project is one among the work stream in Environmental Management Improvement Program Initiative (EMIP) of Oil and Gas organisations. This is of fundamental importance for environmental management plan (EMP). The EMIP road map towards operational performance improvement requires proper EIA to be executed as a preventive measure to reduce and provide full real time transparency in the handling and management of organic and other hydrocarbon generated waste such as drill cuttings and other toxic substances.

Consequently, this has created the need for the assessment and evaluation of the flora biodiversity of the study area as to contribute to the delivery of the EMIP mandates and objectives. On a parallel maxim baseline study ensures that the proposed activities of the TDU project are executed in accordance with statutory requirements. The tropical rainforest constitute the most biological diverse terrestrial ecosystem on earth [11]. It is the predominant natural forest in Nigeria occupying about $10 \%$ of land mass which have been extensively degraded and exist in patches. Across the world, 25 hot-spots have been identified on the basis of species endemism and degree of threat through habitat loss [12]. Forest vegetation represents an important natural resource with diverse scientific, technological, cultural and ecological values at the local, state, national and international levels of societal needs. Quite a number of endemic plant species, which might be of immense economic importance to man and new to science might abound in the study area hence the need for baseline studies prior to the execution of the project. Despite these immense economic and ecological values of forest to man, series of ecological demand by the society are causing significant and irreversible loss to this unique resource [13]. Therefore, in order to assess the levels of future impacts envisaged to emanate due to the TDU project on the ecological biota of the environment, a comprehensive baseline enumeration of flora composition of the area was designed and carried out in wet and dry season. The preparation of the EIA will also support subsequent application and acquisition for Environmental Management Plan (EMP) and Associated Environmental permits for the project.

The proposed baseline study was conducted under the context and premise of reviewing existing literature of the study area including study reports if any, determination and description of the vegetation type, structure and floristic composition of the study zones, determination of key species population and their distribution in terms of phytosociological index of abundance, and develop cost effective mitigation / ameliorative measures, monitoring plans and Environmental Management Plan that meet regulatory requirements. Thus, with the aim of providing and establishing through biological studies appropriate ecologicaldata on the existing status of all identifiable and associated components of vegetation biota of the people and their environ, assessment of the potential positive and negative impact of the proposed project on the environment, proposing appropriate mitigation measures to minimize negative environmental impacts arising from the project and enhance positive impact on the environment, to incorporate the recommendations and fall-out of the EIA process into detailed project design and decisions and suggesting effective environmental management plan. Consequently, developing an environmental management plan (EMP) for all phases of the project development, to give confidence to the planning system by providing for public participation and for consultation processes and to help in the identification of possible alternative processes. Based on the 
above objectives, provide an operation plan as a surveillance network programme to monitor performance and activities of the proposed project.

\section{Materials and Methods}

\subsection{Geographical Description of the Study site / Area}

The study site is located within Eteo Community, Eleme Local Government Area (LGA) of Rivers State, Nigeria. It is located between Lat. $4^{\circ} 45^{\prime} 10^{\prime} \mathrm{N} \& 4^{\circ} 45^{\prime} 20^{\prime \prime} \mathrm{N}$ and Long. $7^{\circ}$ 10 ' 20 "E \& $7^{\circ} 10^{\prime}$ 30"E. (Figs. 1, 2). The study areas are located in the rainforest belt within the equatorial climate region, characterized by high rainfall, high relative humidity and maximum temperature. It is characterized by vegetation complex of primary and secondary structure, heterogenous in nature, with spatial (closed and sparse) and vertical distribution of trees, herbs, shrubs and climbers (lianers) belonging to different families under local environmental conditions and edaphic nature. Such dominant families of plant species include Melastomataceae, Poaceae, Euphorbiaceae, Rubiaceae, Fabaceae, Costaceae, Tiliaceae, Asteraceae, Malvaceae, Cyperaceae, Lamiaceae, Amaranthaceae, Apocynaceae, Verbenaceae, Acanthaceae, and Connaraceae. Others are Convolvulaceae, Arecaceae, Moraceae, Commelinaceae, Smilacaceae, Araceae, Icacinaceae, Vitaceae, Myrtaceae, Selaginellaceae,
Schizacaceae, Adiantaceae, Marattiaceae, Dilleniaceae, Sapindaceae, Anacardiaceae, Polygalaceae, Solanaceae, Asclepiadaceae, Davalliaceae, Cleomaceae, Guttiferae, Passifloraceae, Myristicaceae, Musaceae, Marattiaceae, Burseraceae, Cecropiaceae, Schrophulariaceae, Combretaceae and Loganiaceae. These families among other families in the project area are typical of fallowed secondary vegetation. Geomorphologically, the edaphic and topographic status shows that the site is ecologically characterized by sandy-loam soil.

The environment has witnessed various forms of anthropogenic activities such as farm land / settlement, residential houses, industrial activities and other developmental strives. Though primarily a virgin forest of various vegetation strata, heterogeneous in structures and nature, the effect of these forms of activities resulted to some level of ecological succession which, have consequently left the area with some form of irregular vegetation features and thus can best be categorized as a lowland secondary mosaic forest as described by Hopkin [14] for a low land secondary vegetation system. Despite such ecological succession by anthropogenic influences and natural selection under local environmental condition, the vegetation can still be described as rainforest vegetation in relation to similar view of vegetation analysis by SAF [15] and Edwin -Wosu, [5, 16 - 18].

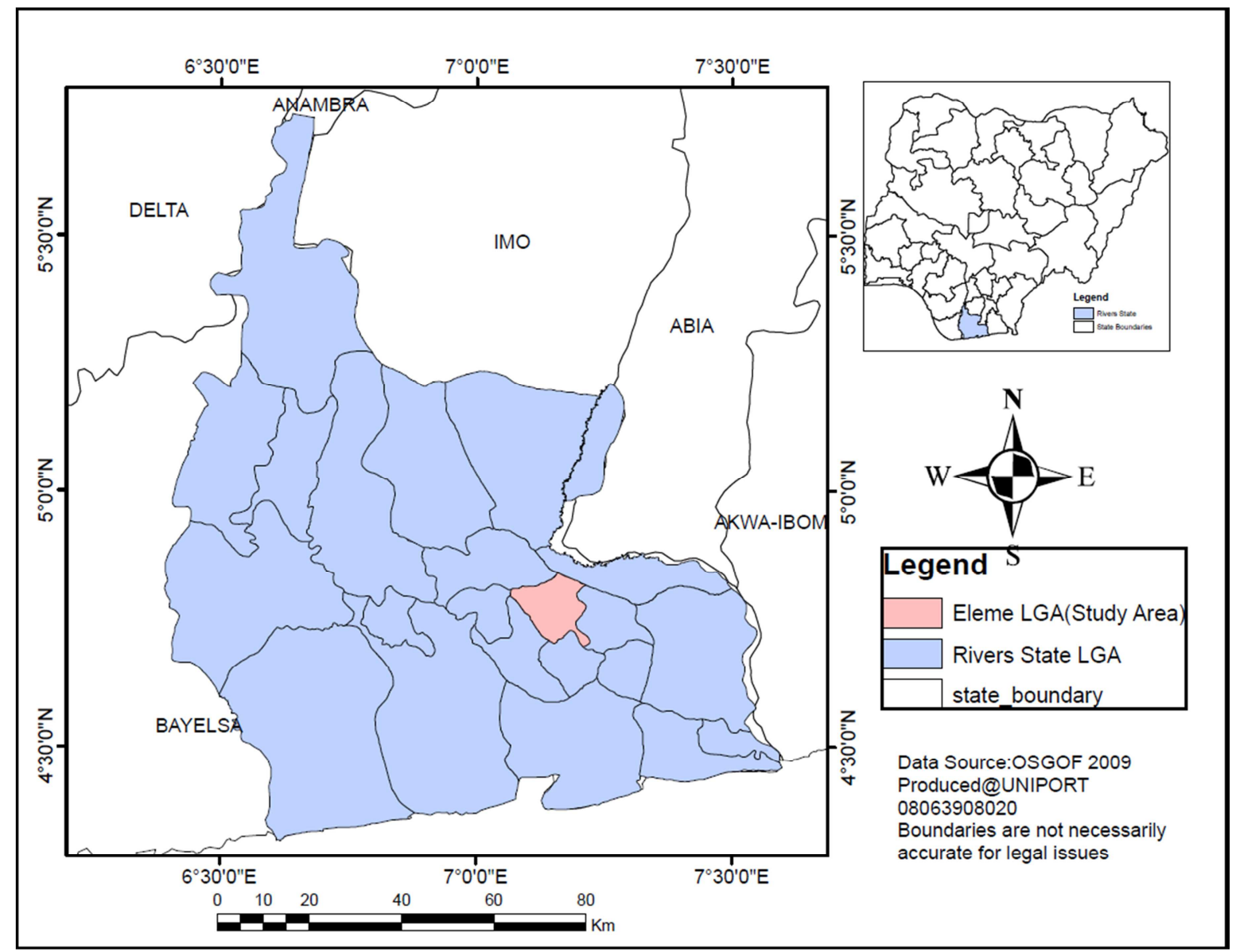

Fig. 1. Rivers State showing Eleme LGA. 


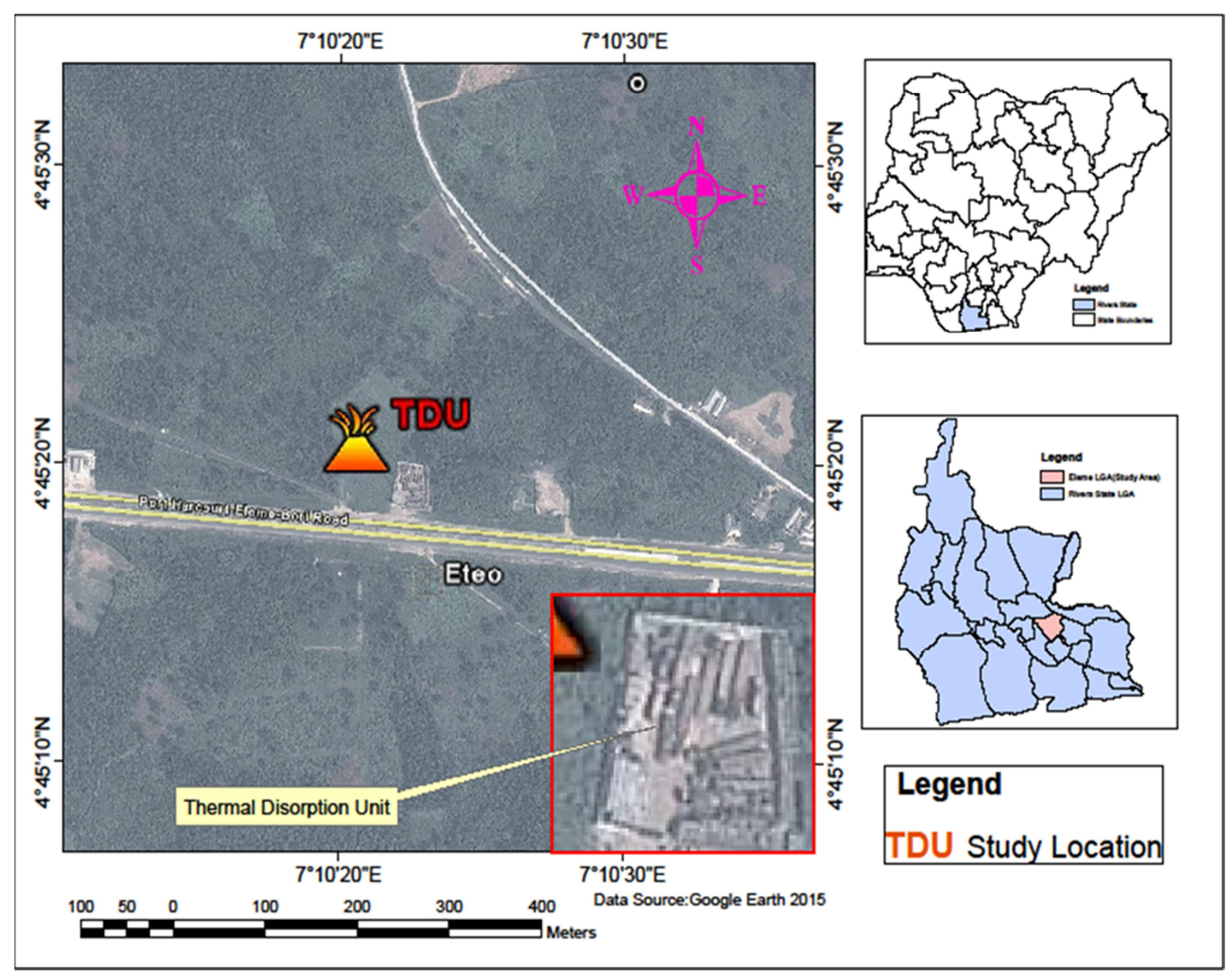

Fig. 2. Study location.

\subsection{Qualitative and Quantitative Flora Assessment}

There are different methods and approaches to environmental impact assessment of development projects. Five major types are commonly used, viz: the checklist, interactive matrices, overlay mapping, network and simulation modeling methods. Based on heterogeneity of the study site and Terms of Reference' (TOR) for the flora biodiversity assessment in line with the irregular physiognomy of the project area, due to human influence, the 'Overlay Mapping method is being adopted using the simple random sampling based on standard procedures for ecological assessment studies [19]. A total of five transect directions in site were systematically sampled. All the important representative plant species sampled were identified in the field as far as possible and were properly authenticated using reference books and Floras such as Burkill, [20 - 24]; Hutchinson and Dalziel, [25-29]; Keay, [30].

\subsection{Data Analysis}

The frequency of distribution, abundance, and density of the most representative species of the area were estimated using the methods of Austin and Greig-Smith [31]; Kershaw [32]; Pryor, [33] and Shukla and Chadel's [34] approach modified in Bonham [35]. The species diversity over the study area was evaluated using the Shannon-Wiener [36] diversity index. Relative density, relative abundance and relative frequency were estimated following Misra [37] method. While the Importance Value Index (IVI) was estimated by adding the values of RD, RA and RF using the Shukla and Chandel [34] method. The ratio of abundance to frequency for different species was determined for distribution patterns. Thus with the "thumb of rule" designated as follows: Regular $(<0.03)$, random $(0.03-0.05)$, and contiguous $(>0.05)$ distribution as adopted by Curtis and Cottam [38]. Similarity index (IS) between the two seasons vegetation complex was derived from the formula given by Sorensen [39].

\section{Result}

Floristic Structure, Composition and Classification

This wet and dry season report on the TDU study area shows that the area is associated with a flora system characterised by secondary vegetation structure in a closed and spatial horizontal continuum. The area has ordinarily been influenced by anthropogenic incursion such as the springing up of built up areas for residential and industrial purposes, domestic fire wood logging for fuel consumption and also 
farmlands by the local inhabitants. The study as observed in Tables 1 and 2 for the TDU operation base had shown that the project sites are richly endowed with floras of various strata and categories belonging to different plant families despite the prevailing influences. The project sites are typical virgin forest though with some levels of secondary heterogeneity as a result of ecological succession incursioned by both natural and human factors. Despite the ecological succession, the study area yet contains plant species in various diversity and abundance with representative species recorded.

Though, there were differences in the ranks of species frequency in different transect, the TDU proposed site in wet season recorded a total representative of 102 species under 39 families in mosaic heterogeneity (Table 1a). Twelve families were prevalently dominant in which 9 families (Euphorbiaceae, Fabaceae, Araceae, Apocynaceae, Malvaceae, Rubiaceae, Passifloraceae, Asteraceae and Cyperaceae) were very abundant while three families (Loganiaceae, Poaceae and Commelinaceae) were in abundance. Among the dominant families, Euphorbiaceae recorded the highest species diversity in terms of richness with 13 species followed by Fabaceae (7 Species), Rubiaceae and Asteraceae had 6 species respectively while Cyperaceae had 5 species. In dry season a total of 59 representative species in 30 families were recorded (Table 2a). Nine families were prevalently dominant with five species (Apocynaceae, Malvaceae, Rubiaceae, Poaceae and Asteraceae)very abundant and four species (Euphorbiaceae, Fabaceae, Tiliaceae and Cyperaceae) in abundance.Among the dominant families Poaceae and Asteraceae were richest with five species each. Under such ecological assessment there was increase in the herbaceous status of habit and life form with the most representative dominant species in the study site as herbs distributed among the 39 and 30 families in wet and dry season respectively.

Phytosociological assessment of species prevalence around the TDU proposed site shows that among the representative species (Table 1b) in wet season, 18 species had the highest frequency occurrence of $100=1.61 \%$ while the density showed 49 species with maximum range value of $10-60=$ $0.80-4.80 \%$ with the highest density value of $60=4.80 \%$ recorded with Manihot esculenta (cassava). A total of 76 species had maximum abundance with a range value of 10 $100(0.50-4.98 \%)$ in which Urena lobata and Oldenlandia herbacearecorded the highest abundance values of $100=$ $4.98 \%$ respectively, while Oldenlandia herbaceahave shown dominance with Important Value Index of $8.82 \%$. Species diversity is one of the major criteria in recognising the importance of an area for conservation priority. The diversity of species in the study area has shown some degree of variation in flora richness and evenness among representative samples. Result showed that cassava and Oldenlandia herbacea recorded the highest diversity with Index Value of 0.05 richness and 0.02 evenness respectively among representative with maximum value range of $0.04-0.05$ richness and 0.02 evenness. The ratio of abundance to frequency showed that the species of the study area were mostly contiguous in distribution pattern with a spatial continuum of $98(96.08 \%)$ species while $3(2.94 \%)$ and 1 $(1.0 \%)$ were regularly and randomly distributed respectively (Fig. 3). Generally, in all bounding coordinate within the study site there was increase in herbaceous status in terms of habit with a representative total of $54(52.94 \%)$ species as herbs, 38 (37.26\%) as shrubs and $10(9.8 \%)$ as trees (Fig. 4).

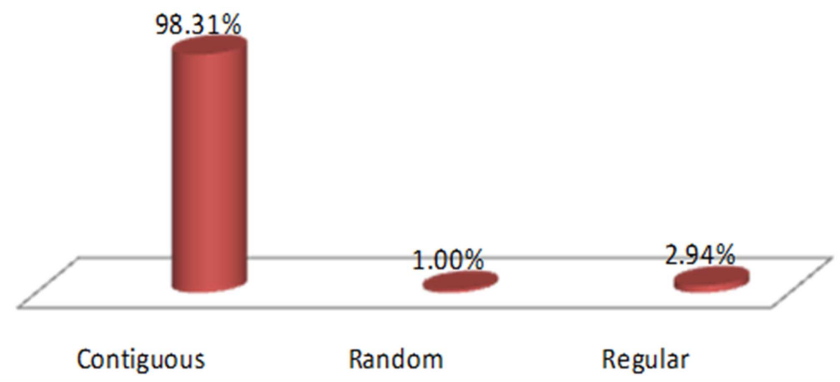

Fig. 3. Wet season distribution pattern of species.

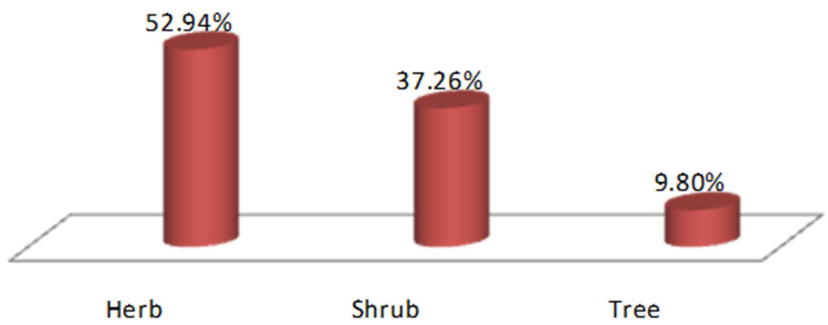

Fig. 4. Wet season life form of species.

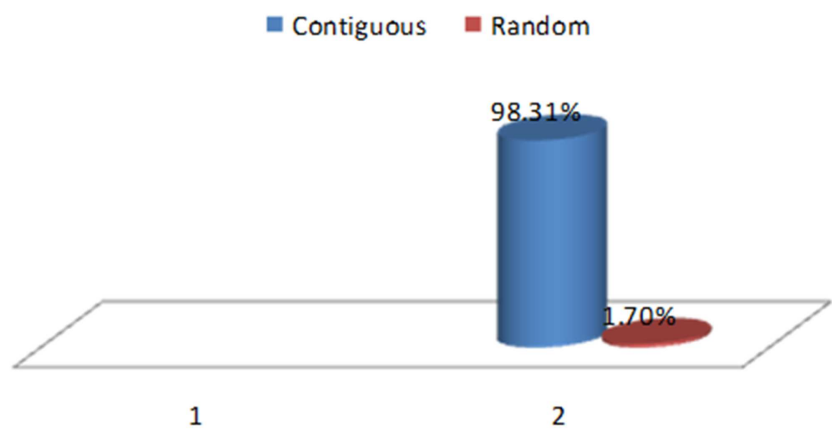

Fig. 5. Dry season distribution pattern of species

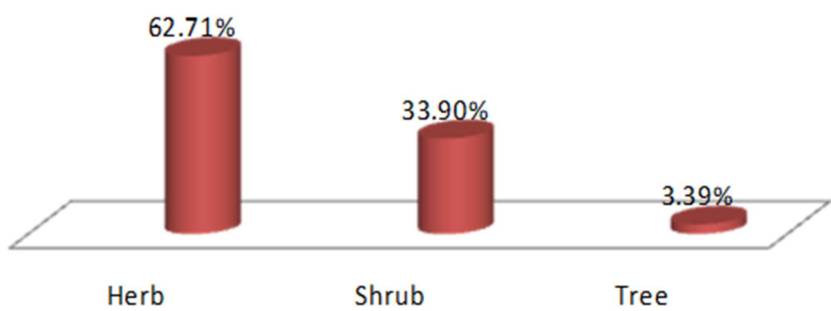

Fig. 6. Dry season life form of species.

In dry season one species ( $H$. rotundifolia) had the highest frequency of $100=5.05 \%$, while 18 species recorded maximum density range of $10-40=2.04-1.22 \%$ with carpet grass having the highest density. Fourty one species had maximum abundance range of $10-80=0.90-7.23 \%$ with $P$. indica having the highest abundance of $80=7.23 \%$. The 
highest IVI of $16.71 \%$ was recorded by carpet grass, with highest diversity of 0.07 richness and 0.04 evenness. The distribution pattern had $58(98.31 \%)$ species with contiguous and one $(1.70 \%)$ as random distribution (Fig. 5), while life form habit had 37 (62.71\%) as herbs, 20 (33. 90\%) as shrubs and $2(3.39 \%)$ as trees (Table 2b) (Fig. 6). Despite the variation in season, on the basis of similarity in species composition of the vegetation structure the site recorded maximum similarity of $56 \%$.

Generally, the plant species in the project location were not remarkably infected with pathogens when compared to the apparent observation of leaf sports, die back, leaf blotch, dry rot, stem rot, stem corky and fungi and mushroom infestation as at the period of sampling.

Table 1A. Wet season Survey Estimate of Representative Species around the TDU proposed site.

\begin{tabular}{|c|c|c|c|c|c|c|}
\hline $\mathbf{S} / \mathbf{N}$ & Species & $\% \mathrm{~F}$ & Family & Habit & Common Name & Remark \\
\hline 1 & Alchornea cordifolia (Schum \& Thonn) Mull - Arg. & 80 & Euphorbiaceae & Shrub & Christmas bush & ++++ \\
\hline 2 & Mallotus subulatus Mull - Arg. & 100 & Euphorbiaceae & Shrub & Kamala plant & +++++ \\
\hline 3 & Mallotus oppositifolus (Geisel) Mull - Arg & 60 & Euphorbiaceae & Shrub & Kamala plant & +++ \\
\hline 4 & Cnestis ferruginea DC & 40 & Connaraceae & Shrub & NA & ++ \\
\hline 5 & Smilax anceps Willd. & 40 & Smilacaceae & Herb & West African sarsaparilla & ++ \\
\hline 6 & Anthonotha macrophylla P. Beauv. & 100 & Fabaceae-caesal. & Shrub & West African rose wood & +++++ \\
\hline 7 & Anthonotha obanensis (Bak.f.) J. Leonard & 100 & Fabaceae-caesal. & Shrub & West African rose wood & +++++ \\
\hline 8 & Anchormanes difformis (B1.) Engl. & 80 & Araceae & Shrub & Forest Anchormanes & ++++ \\
\hline 9 & Milletia aboensis (Hook. f.) Bak. & 60 & Fabaceae & Shrub & Fermentation plant & +++ \\
\hline 10 & Icacina trichantha Oliv. & 60 & Icacinaceae & Herb & NA & +++ \\
\hline 11 & Voacanga africana Stapf. & 80 & Apocynaceae & Shrub & NA & ++++ \\
\hline 12 & Urena lobata Linn. & 20 & Malvaceae & Shrub & NA & + \\
\hline 13 & Oldenlandia corymbosa Linn. & 60 & Rubiaceae & Herb & Mille - graines & +++ \\
\hline 15 & Barteria nigritiana Hook. f. & 80 & Passifloraceae & Shrub & NA & ++++ \\
\hline 16 & Elaeis guineensis Jacq & 100 & Arecaceae & Tree & Oil palm tree & +++++ \\
\hline 17 & Anthocleista vogelii Planch. & 80 & Loganiaceae & Tree & Cabbage tree & ++++ \\
\hline 18 & Anthocleista djalonesis A. Chev. & 80 & Loganiaceae & Tree & Cabbage tree & ++++ \\
\hline 19 & Harrungana madagascariensis Ham ex Pour & 100 & Guttiferae & Shrub & Dragon's blood & +++++ \\
\hline 20 & Rauvolfia vormitora Afzel. & 80 & Apocynaceae & Shrub & Swizzle stick & ++++ \\
\hline 21 & Bambusa vulgaris Schrad. & 60 & Poaceae & Tree & Indian bamboo & +++ \\
\hline 22 & Alchornea laxiflora (Benth.) Pax \& K. Hoffm. & 80 & Euphorbiaceae & Shrub & Christmas bush & ++++ \\
\hline 23 & Manniophyton fulvum Mull - Arg. & 20 & Euphorbiaceae & Shrub & NA & + \\
\hline 24 & Hibiscus surratensis Linn. & 20 & Malvaceae & Herb & Bush sorrel & + \\
\hline 25 & Palisota hirsuta (Thunb.) K. Schum. & 80 & Commelinaceae & Shrub & NA & ++++ \\
\hline 26 & Palisota ambigua (P. Beauv.) C.B. Cl. & 60 & Commelinaceae & Shrub & NA & +++ \\
\hline 27 & Costus lucanusianus J. Braun \& K. Schum. & 80 & Costaceae & Shrub & Ginger lily & ++++ \\
\hline 30 & Vitex doniana Sweet. & 20 & Vitaceae & Shrub & Black plum & + \\
\hline 31 & Funfumia africana (Benth.) Stapf. & 60 & Apocynaceae & Shrub & NA & +++ \\
\hline 32 & Anchormanes giganteus Engl. & 80 & Araceae & Herb & Giant Anchormanes & ++++ \\
\hline 33 & Psiduim guajava Linn & 60 & Myrtceae & Shrub & Guava & +++ \\
\hline 34 & Maesobotrya barteri (Baill) Hutch. & 40 & Euphorbiaceae & Shrub & Bush cherry & ++ \\
\hline 35 & Grifformia physocarpa Baill. & 40 & Fabaceae & Tree & NA & ++ \\
\hline 36 & Selaginella myosurus (SW.) Alston. & 100 & Selaginellaceae & Herb & Fern & +++++ \\
\hline 37 & Lygodium macrophyllum & 100 & Schizacaceae & Herb & Fern & +++++ \\
\hline 38. & Senna hirsuta (Linn.) Irwin \& Barneby & 80 & Fabaceae-ceasal & Shrub & Senna & ++++ \\
\hline 39 & Manihot esculenta Crantz & 100 & Euphorbiaceae & Shrub & Cassava & +++++ \\
\hline 40 & Pteris acanthonura & 20 & Adiantaceae & Herb & Fern & + \\
\hline 41 & Cissus aralioides (Welw.) Planch. & 60 & Vitaceae & Herb & NA & +++ \\
\hline 42 & Stachytarpheta cayennensis (LC Rich.) Schau. & 80 & Verbenaceae & Shrub & Rat tail & ++++ \\
\hline 43 & Melastomastrum capitatum (Vahl.) A \& R. Fernandes & 40 & Melastomataceae & Shrub & NA & ++ \\
\hline 44 & Antidesma vogelianum Mull- Arg. & 40 & Euphorbiaceae & Shrub & NA & ++ \\
\hline 45 & Glyphea brevis (Spreng) Monachino & 40 & Tiliaceae & Shrub & NA & ++ \\
\hline 46 & Craterispernum cerrunanthum Hiern. & 60 & Rubiaceae & Shrub & NA & +++ \\
\hline 47 & Craterispernum caudatum & 60 & Rubiaceae & Shrub & NA & +++ \\
\hline
\end{tabular}




\begin{tabular}{|c|c|c|c|c|c|c|}
\hline $\mathbf{S} / \mathbf{N}$ & Species & $\% \mathrm{~F}$ & Family & Habit & Common Name & Remark \\
\hline 48 & Macaranga barteri Mull - Arg. & 80 & Euphorbiaceae & Shrub & NA & ++++ \\
\hline 49 & Macaranga spinosa Mull - Arg. & 80 & Euphorbiaceae & Shrub & NA & ++++ \\
\hline 50 & Bridella ferruginea Benth. & 60 & Euphorbiaceae & Shrub & NA & +++ \\
\hline 51 & Bridella grandis Pierre & 60 & Euphorbiaceae & Tree & NA & +++ \\
\hline 52 & Paullina pinnata Linn. & 100 & Sapindaceae & Herb & Bread \& Cheese & +++++ \\
\hline 53 & Tetracera alnifolia & 20 & Dilleniaceae & Herb & Liane cord & + \\
\hline 54 & Spondias mombin Linn. & 40 & Anarcardiaceae & Tree & Hog plum & ++ \\
\hline 55 & Rhektophyllum mrabile N.E. Br. & 60 & Araceae & Herb & Bush cocoyam & +++ \\
\hline 56 & Smeathmania pubescens Soland. & 40 & Passifloraceae & Tree & NA & ++ \\
\hline 57 & Carpoloba leutea G.Don. & 20 & Polygalaceae & Shrub & Poor man's candle & + \\
\hline 58 & Physallis angulata Linn & 40 & Solanaceae & Herb & Angular water cherry & ++ \\
\hline 59 & Triumfetta cordifolia A.Rich. & 100 & Tiliaceae & Herb & Bur weed & +++++ \\
\hline 60 & Ipomoea involucrata P. Beauv. & 20 & Convolvulaceae & Herb & Morning glory & + \\
\hline 61 & Landolphia dulcis (R. Br) Pichon & 20 & Apocynaceae & Herb & Rubber vine & + \\
\hline 62 & Stachytarpheta jamaicensis Vahl. & 60 & Verbenaceae & Shrub & Brazilian tea & +++ \\
\hline 63 & Albizia leebeck (Linn.) Benth. & 40 & Fabaceae - mimo & Tree & Women tongue & ++ \\
\hline 64 & Pergularia daemia (Forssk.) Chiov. & 20 & Asclepiadaceae & Herb & NA & + \\
\hline 65 & Adenia labata (Jacq) Engl. & 20 & Passifloraceae & Herb & NA & + \\
\hline 66 & Adenia caesampeloides (Planch) Harms & 40 & Passifloraceae & Herb & NA & ++ \\
\hline 67 & Lonchocarpus sericeus (Poir) HB \& K & 20 & Fabaceae - papi & Shrub & Cube root & + \\
\hline 68 & Mussaenda landolphioides Wernham & 40 & Rubiaceae & Herb & NA & ++ \\
\hline 69 & Marattia fraxinea & 100 & Marattiaceae & Herb & Fern & +++++ \\
\hline 70 & Ficus exasperata Vahl. & 20 & Moraceae & Shrub & Sand paper plant & + \\
\hline 71 & Panicum maximum Jacq & 100 & Poaceae & Herb & Guinea grass & +++++ \\
\hline 72 & Aspilla bussei O. Hoffm \& Muschl & 100 & Asteraceae & Herb & White hemorrhage & +++++ \\
\hline 73 & Sida rhombifolia Linn. & 100 & Malvaceae & Herb & Wire weed & +++++ \\
\hline 74 & Sida garkaena Polak & 100 & Malvaceae & Herb & Broom weed & +++++ \\
\hline 75 & Conyzia summatrensis (Retz) Walker & 20 & Asteraceae & Herb & Fleabane & + \\
\hline 76 & Nephrolepis pumicola & 100 & Davalliaceae & Herb & Fern & +++++ \\
\hline 77 & Nephrolepis bisserata & 100 & Davalliaceae & Herb & Fern & +++++ \\
\hline 78 & Cleom rotidosperma $\mathrm{DC}$. & 40 & Cleomaceae & Herb & Spider flower & ++ \\
\hline 79 & Chromotaena odorata (Linn) RM King \& Robinson & 40 & Asteraceae & Herb & Siam weed & ++ \\
\hline 80 & Aspilla africana (Pers) CD Adams. & 60 & Asteraceae & Herb & Heamorrhage plant & +++ \\
\hline 81 & Ageratum conyziodes Linn. & 60 & Asteraceae & Herb & Goat weed & +++ \\
\hline 82 & Solenostemon monostachyus (P. Beauv.) Brig. & 20 & Lamiaceae & Herb & NA & + \\
\hline 83 & Phyllanthus amarus Schum \& Thonn. & 60 & Euphorbiaceae & Herb & Chanca & +++ \\
\hline 84 & Spermaccoce ocymoides Burm f. & 40 & Rubiaceae & Herb & False button weed & ++ \\
\hline 85 & Heterotis rotundifolia (Sm.) Jac. Fel. & 100 & Melastomataceae & Herb & Cheek weed & +++++ \\
\hline 86 & Cyathula prostrata (L.) Blums. & 80 & Amaranthaceae & Herb & Cyathule & ++++ \\
\hline 87 & Archyranthes aspera Linn. & 60 & Acanthaceae & Herb & Devil horsewhip & +++ \\
\hline 88 & Lapistemon owariensis (P. Beauv.) Hallier .f. & 40 & Covolvulaceae & Herb & NA & ++ \\
\hline 89 & Plastosma africanum P. Beauv. & 60 & Lamiaceae & Herb & NA & +++ \\
\hline 90 & Spillanthes filicaulis (Schum \& Thonn.) CD Adams & 80 & Asteraceae & Herb & Brazil cress & ++++ \\
\hline 91 & Mariscus longbreateatus Cherm. & 40 & Cyperaceae & Herb & Sedge & ++ \\
\hline 92 & Mariscus flabelliformis Kunth. & 40 & Cyperaceae & Herb & Sedge & ++ \\
\hline 93 & Kyllinga bulbosa Beauv, & 60 & Cyperaceae & Herb & Sedge & +++ \\
\hline 94 & Asystasia gagentica (Linn.) T. Anders. & 60 & Acanthaceae & Herb & NA & +++ \\
\hline 95 & Alternanthera sessilis (Linn.) DC. & 80 & Amarantheceae & Herb & Sessile joyweed & ++++ \\
\hline 96 & Anchormanes giganteus Engl. & 80 & Araceae & Herb & Giant Anchormanes & ++++ \\
\hline 97 & Aneilema beniniense (P. Beauv.) Kunth & 80 & Commelinaceae & Herb & NA & ++++ \\
\hline 98 & Mariscus alternifolius Vahl. & 60 & Cyperaceae & Herb & Sedge & +++ \\
\hline 99 & Perotis indica (Linn.) O. Ktze & 80 & Poaceae & Herb & Grass & ++++ \\
\hline 100 & Oplimenus baumanii (Retz) P. Beauv. & 80 & Poaceae & Herb & Grass & ++++ \\
\hline 101 & Oldenlandia herbacea (Linn.) Roxb & 40 & Rubiaceae & Herb & NA & ++ \\
\hline 102 & Cypenes rotundus Linn. & 40 & Cyperaceae & Herb & Purple nutsedge & ++ \\
\hline
\end{tabular}

Note: $+(1-25)$ Very scarce, ++ (26-59) Scarce, $+++(60-79)$ Abundant, $++++>(80-\alpha)$ Very abundant, NA- Not available, \%F- Percentage frequency. 
16 Nsirim L. Edwin-Wosu and Victor N. Sunday: A Baseline Environmental Impact Assessment (EIA) of Proposed Sit for Thermal Desorption Unit (TDU) Waste Management Project in Rivers State, Nigeria: Floristic Composition Approach

Table 1B. Wet Season Quantitative List of Representative Species around the TDU proposed site.

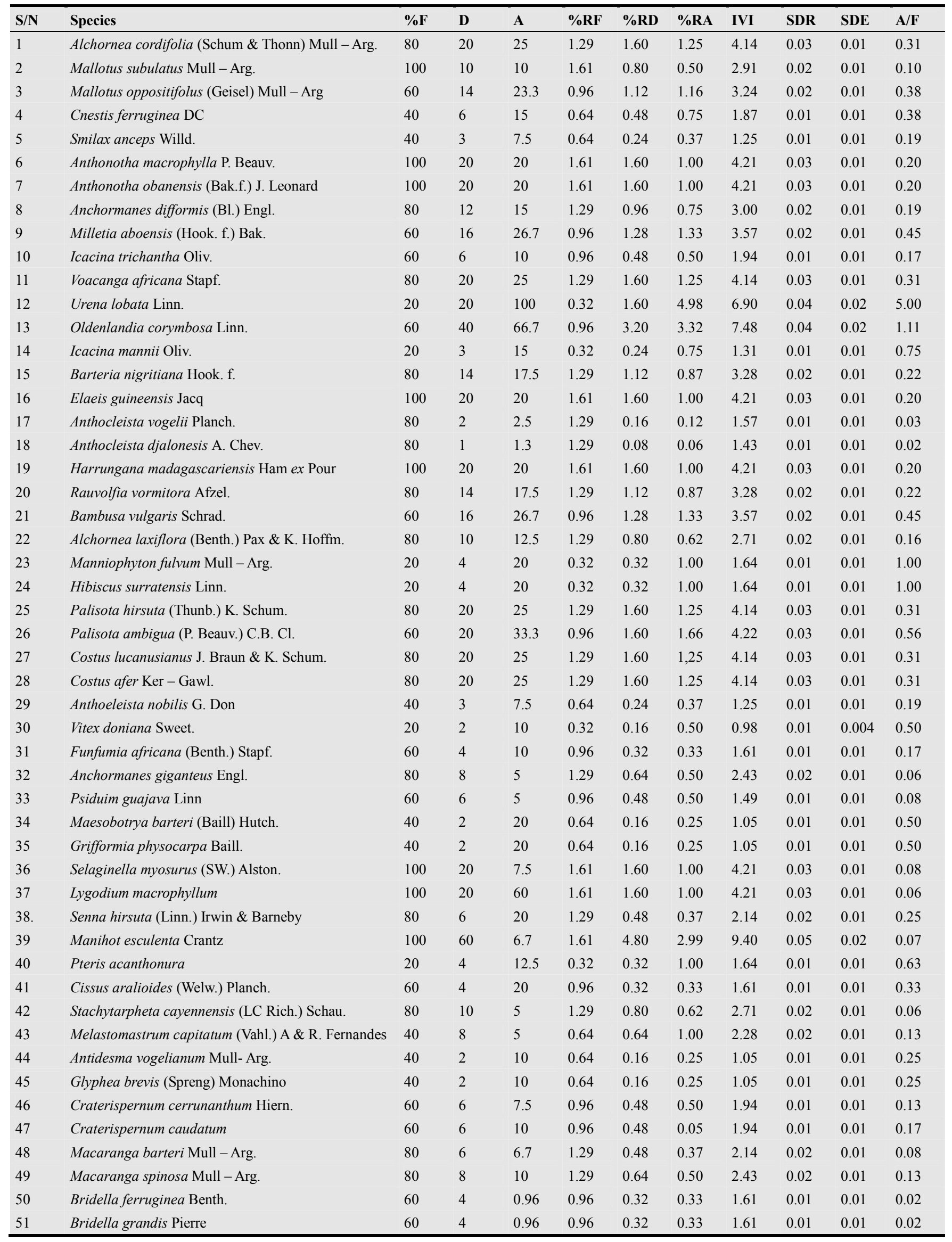




\begin{tabular}{|c|c|c|c|c|c|c|c|c|c|c|c|}
\hline $\mathbf{S} / \mathbf{N}$ & Species & $\% \mathbf{F}$ & D & $\mathbf{A}$ & $\%$ RF & $\%$ RD & $\% \mathbf{R A}$ & IVI & SDR & SDE & $\mathbf{A} / \mathbf{F}$ \\
\hline 52 & Paullina pinnata Linn. & 100 & 12 & 12 & 1.61 & 0.96 & 0.60 & 3.17 & 0.02 & 0.01 & 0.12 \\
\hline 53 & Tetracera alnifolia & 20 & 2 & 10 & 0.32 & 0.16 & 0.50 & 0.98 & 0.01 & 0.004 & 0.50 \\
\hline 54 & Spondias mombin Linn. & 40 & 1 & 2.5 & 0.64 & 0.08 & 0.12 & 0.84 & 0.01 & 0.004 & 0.06 \\
\hline 55 & Rhektophyllum mrabile N.E. Br. & 60 & 4 & 6.7 & 0.96 & 0.32 & 0.33 & 1.61 & 0.01 & 0.01 & 0.11 \\
\hline 56 & Smeathmania pubescens Soland. & 40 & 2 & 5 & 0.64 & 0.16 & 0.25 & 1.05 & 0.01 & 0.01 & 0.13 \\
\hline 57 & Carpoloba leutea G.Don. & 20 & 1 & 5 & 0.32 & 0.08 & 0.25 & 0.65 & 0.01 & 0.003 & 0.25 \\
\hline 58 & Physallis angulata Linn & 40 & 2 & 5 & 0.64 & 0.16 & 0.25 & 1.05 & 0.01 & 0.01 & 0.13 \\
\hline 59 & Triumfetta cordifolia A.Rich. & 100 & 6 & 6 & 1.61 & 0.48 & 0.30 & 2.39 & 0.02 & 0.01 & 0.06 \\
\hline 60 & Ipomoea involucrata P. Beauv. & 20 & 2 & 10 & 0.32 & 0.16 & 0.50 & 0.98 & 0.01 & 0.004 & 0.50 \\
\hline 61 & Landolphia dulcis (R. Br) Pichon & 20 & 2 & 10 & 0.32 & 0.16 & 0.50 & 0.98 & 0.01 & 0.004 & 0.50 \\
\hline 62 & Stachytarpheta jamaicensis Vahl. & 60 & 6 & 10 & 0.96 & 0.48 & 0.50 & 1.94 & 0.01 & 0.01 & 0.10 \\
\hline 63 & Albizia leebeck (Linn.) Benth. & 40 & 2 & 5 & 0.64 & 0.16 & 0.25 & 1.05 & 0.01 & 0.01 & 0.13 \\
\hline 64 & Pergularia daemia (Forssk.) Chiov. & 20 & 1 & 5 & 0.32 & 0.08 & 0.25 & 0.65 & 0.01 & 0.01 & 0.25 \\
\hline 65 & Adenia labata (Jacq) Engl. & 20 & 1 & 5 & 0.32 & 0.08 & 0.25 & 0.65 & 0.01 & 0.003 & 0.25 \\
\hline 66 & Adenia caesampeloides (Planch) Harms & 40 & 1 & 2.5 & 0.64 & 0.08 & 0.12 & 0.84 & 0.01 & 0.004 & 0.06 \\
\hline 67 & Lonchocarpus sericeus (Poir) HB \& K & 20 & 2 & 10 & 0.32 & 0.16 & 0.50 & 0.98 & 0.01 & 0.004 & 0.50 \\
\hline 68 & Mussaenda landolphioides Wernham & 40 & 4 & 10 & 0.64 & 0.32 & 0.50 & 1.46 & 0.01 & 0.01 & 0.25 \\
\hline 69 & Marattia fraxinea & 100 & 20 & 20 & 1.61 & 1.6 & 1.00 & 4.21 & 0.03 & 0.01 & 0.20 \\
\hline 70 & Ficus exasperata Vahl. & 20 & 1 & 5 & 0.32 & 0.08 & 0.25 & 0.65 & 0.01 & 0.003 & 0.25 \\
\hline 71 & Panicum maximum Jacq & 100 & 40 & 40 & 1.61 & 3.20 & 1.99 & 6.80 & 0.04 & 0.02 & 0.40 \\
\hline 72 & Aspilla bussei O. Hoffm \& Muschl & 100 & 20 & 20 & 1.61 & 1.60 & 1.00 & 4.21 & 0.03 & 0.01 & 0.20 \\
\hline 73 & Sida rhombifolia Linn. & 100 & 20 & 20 & 1.61 & 1.60 & 1.00 & 4.21 & 0.03 & 0.01 & 0.20 \\
\hline 74 & Sida garkaena Polak & 100 & 20 & 20 & 1.61 & 1.60 & 1.00 & 4.21 & 0.03 & 0.01 & 0.20 \\
\hline 75 & Conyzia summatrensis (Retz) Walker & 20 & 2 & 10 & 0.32 & 0.16 & 0.50 & 0.98 & 0.01 & 0.004 & 0.50 \\
\hline 76 & Nephrolepis pumicola & 100 & 20 & 20 & 1.61 & 1.60 & 1.00 & 4.21 & 0.03 & 0.01 & 0.20 \\
\hline 77 & Nephrolepis bisserata & 100 & 24 & 24 & 1.61 & 1.92 & 1.20 & 4.73 & 0.03 & 0.02 & 0.24 \\
\hline 78 & Cleom rotidosperma $\mathrm{DC}$. & 40 & 10 & 25 & 0.64 & 0.80 & 1,25 & 2.69 & 0.02 & 0.01 & 0.63 \\
\hline 79 & Chromotaena odorata (Linn) RM King \& Robinson & 40 & 20 & 50 & 0.64 & 1.60 & 2.49 & 4.73 & 0.03 & 0.02 & 1.25 \\
\hline 80 & Aspilla africana (Pers) CD Adams. & 60 & 40 & 66.7 & 0.96 & 3.20 & 3.32 & 7.48 & 0.04 & 0.02 & 1.11 \\
\hline 81 & Ageratum conyziodes Linn. & 60 & 10 & 16.7 & 0.96 & 0.80 & 0.83 & 2.59 & 0.02 & 0.01 & 0.28 \\
\hline 82 & Solenostemon monostachyus (P. Beauv.) Brig. & 20 & 6 & 30 & 0.32 & 0.48 & 1.50 & 2.30 & 0.02 & 0.01 & 1.50 \\
\hline 83 & Phyllanthus amarus Schum \& Thonn. & 60 & 20 & 33.3 & 0.96 & 1.60 & 1.66 & 4.22 & 0.03 & 0.01 & 0.56 \\
\hline 84 & Spermaccoce ocymoides Burm f. & 40 & 12 & 30 & 0.64 & 0.96 & 1.50 & 3.10 & 0.02 & 0.01 & 0.75 \\
\hline 85 & Heterotis rotundifolia (Sm.) Jac. Fel. & 100 & 20 & 20 & 1.61 & 1.60 & 1.00 & 4.21 & 0.03 & 0.01 & 0.20 \\
\hline 86 & Cyathula prostrata (L.) Blums. & 80 & 16 & 20 & 1.29 & 1.28 & 1.00 & 3.57 & 0.02 & 0.01 & 0.25 \\
\hline 87 & Archyranthes aspera Linn. & 60 & 20 & 33.3 & 0.96 & 1.60 & 1.66 & 4.22 & 0.03 & 0.01 & 0.56 \\
\hline 88 & Lapistemon owariensis (P. Beauv.) Hallier .f. & 40 & 6 & 15 & 0.64 & 0.48 & 0.75 & 1.87 & 0.01 & 0.01 & 0.38 \\
\hline 89 & Plastosma africanum P. Beauv. & 60 & 8 & 13.3 & 0.96 & 0.64 & 0.66 & 2.26 & 0.02 & 0.01 & 0.22 \\
\hline 90 & Spillanthes filicaulis (Schum \& Thonn.) CD Adams & 80 & 20 & 25 & 1.29 & 1.60 & 1.25 & 4.14 & 0.03 & 0.01 & 0.31 \\
\hline 91 & Mariscus longbreateatus Cherm. & 40 & 8 & 20 & 0.64 & 0.64 & 1.00 & 2.28 & 0.02 & 0.01 & 0.50 \\
\hline 92 & Mariscus flabelliformis Kunth. & 40 & 8 & 20 & 0.64 & 0.64 & 1.00 & 2.28 & 0.02 & 0.01 & 0.50 \\
\hline 93 & Kyllinga bulbosa Beauv, & 60 & 12 & 20 & 0.96 & 0.96 & 1.00 & 2.92 & 0.02 & 0.01 & 0.33 \\
\hline 94 & Asystasia gagentica (Linn.) T. Anders. & 60 & 6 & 10 & 0.96 & 0.48 & 0.50 & 1.94 & 0.01 & 0.01 & 0.17 \\
\hline 95 & Alternanthera sessilis (Linn.) DC. & 80 & 20 & 25 & 1.29 & 1.60 & 1.25 & 4.14 & 0.03 & 0.01 & 0.31 \\
\hline 96 & Anchormanes giganteus Engl. & 80 & 6 & 7.5 & 1.29 & 0.48 & 0.37 & 2.14 & 0.02 & 0.01 & 0.08 \\
\hline 97 & Aneilema beniniense (P. Beauv.) Kunth & 80 & 12 & 15 & 1.29 & 0.96 & 0.75 & 3.00 & 0.02 & 0.01 & 0.19 \\
\hline 98 & Mariscus alternifolius Vahl. & 60 & 20 & 33.3 & 0.96 & 1.60 & 1.66 & 4.22 & 0.03 & 0.01 & 0.56 \\
\hline 99 & Perotis indica (Linn.) O. Ktze & 80 & 40 & 50 & 1.29 & 3.20 & 2.49 & 6.98 & 0.04 & 0.02 & 0.63 \\
\hline 100 & Oplimenus baumanii (Retz) P. Beauv. & 80 & 40 & 50 & 1.29 & 3.20 & 2.49 & 6.98 & 0.04 & 0.02 & 0.63 \\
\hline 101 & Oldenlandia herbacea (Linn.) Roxb & 40 & 40 & 100 & 0.64 & 3.20 & 4.98 & 8.82 & 0.05 & 0.02 & 2.50 \\
\hline \multirow[t]{2}{*}{102} & Cypenes rotundus Linn. & 40 & 20 & 50 & 0.64 & 1.60 & 2.49 & 4.73 & 0.03 & 0.02 & 1.25 \\
\hline & & 6220 & 1250 & 2006.1 & & & & 299.92 & & & \\
\hline
\end{tabular}


18 Nsirim L. Edwin-Wosu and Victor N. Sunday: A Baseline Environmental Impact Assessment (EIA) of Proposed Sit for Thermal Desorption Unit (TDU) Waste Management Project in Rivers State, Nigeria: Floristic Composition Approach

Table 2A. Dry Season Survey Estimate of Representative Species around the TDU proposed site.

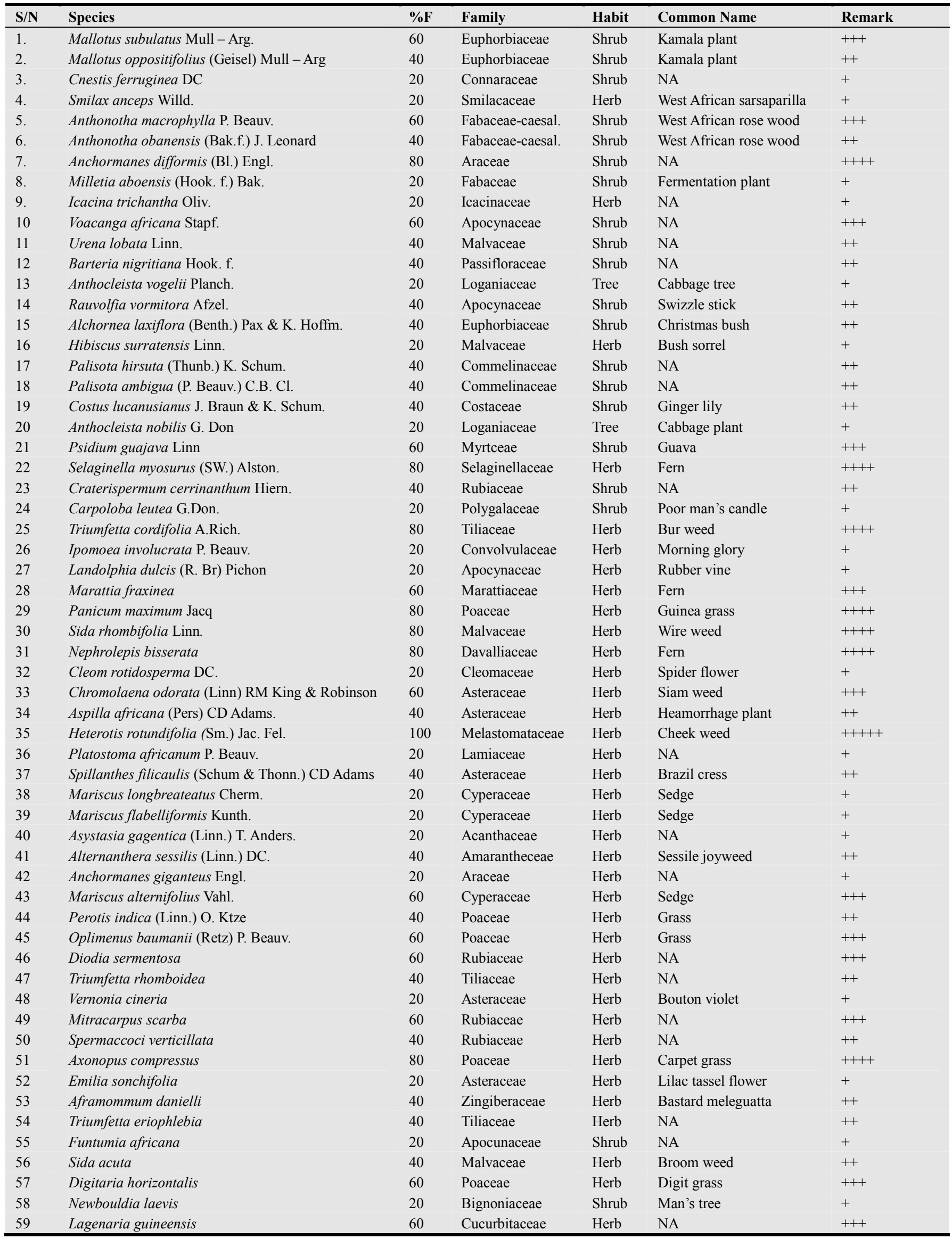


Table 2B. Dry Season Quantitative List of Representative Species around the TDU proposed site.

\begin{tabular}{|c|c|c|c|c|c|c|c|c|c|c|c|}
\hline $\mathbf{S} / \mathbf{N}$ & Species & $\% \mathrm{~F}$ & $\mathbf{D}$ & $\mathbf{A}$ & $\%$ RF & $\%$ RD & $\%$ RA & IVI & SDR & SDE & $\mathbf{A} / \mathbf{F}$ \\
\hline 1 & Mallotus subulatus Mull - Arg. & 60 & 4 & 6.7 & 3.03 & 0.82 & 0.61 & 4.46 & 0.03 & 0.02 & 0.11 \\
\hline 2 & Mallotus oppositifolius (Geisel) Mull - Arg & 40 & 6 & 15 & 2.02 & 1.22 & 1.36 & 4.60 & 0.03 & 0.02 & 0.38 \\
\hline 3 & Cnestis ferruginea DC & 20 & 2 & 10 & 1.01 & 0.41 & 0.90 & 2.32 & 0.02 & 0.01 & 0.50 \\
\hline 4 & Smilax anceps Willd. & 20 & 2 & 10 & 1.01 & 0.41 & 0.90 & 2.32 & 0.02 & 0.01 & 0.50 \\
\hline 5 & Anthonotha macrophylla P. Beauv. & 60 & 6 & 10 & 3.03 & 1.22 & 0.90 & 5.15 & 0.03 & 0.02 & 0.17 \\
\hline 6 & Anthonotha obanensis (Bak.f.) J. Leonard & 40 & 3 & 7.5 & 2.02 & 0.61 & 0.68 & 3.31 & 0.02 & 0.01 & 0.19 \\
\hline 7 & Anchormanes difformis (B1.) Engl. & 80 & 2 & 2.5 & 4.04 & 0.41 & 0.23 & 4.68 & 0.03 & 0.02 & 0.03 \\
\hline 8 & Milletia aboensis (Hook. f.) Bak. & 20 & 7 & 35 & 1.01 & 1.43 & 3.16 & 5.60 & 0.03 & 0.02 & 1.75 \\
\hline 9 & Icacina trichantha Oliv. & 20 & 1.6 & 8 & 1.01 & 0.33 & 0.72 & 2.06 & 0.01 & 0.01 & 0.40 \\
\hline 10 & Voacanga africana Stapf. & 60 & 4 & 6.7 & 3.03 & 0.82 & 0.61 & 4.46 & 0.03 & 0.02 & 0.11 \\
\hline 11 & Urena lobata Linn. & 40 & 2 & 5 & 2.02 & 0.41 & 0.45 & 2.88 & 0.02 & 0.01 & 0.13 \\
\hline 12 & Barteria nigritiana Hook. $\mathrm{f}$. & 40 & 5 & 12.5 & 2.02 & 1.02 & 1.13 & 4.17 & 0.02 & 0.01 & 0.31 \\
\hline 13 & Anthocleista vogelii Planch. & 20 & 1 & 5 & 1.01 & 0.20 & 0.45 & 1.66 & 0.01 & 0.01 & 0.25 \\
\hline 14 & Rauvolfia vormitora Afzel. & 40 & 3 & 7.5 & 2.02 & 0.61 & 0.68 & 3.31 & 0.003 & 0.002 & 0.19 \\
\hline 16 & Hibiscus surratensis Linn. & 20 & 2 & 10 & 1.01 & 0.41 & 0.90 & 2.32 & 0.02 & 0.01 & 0.50 \\
\hline 17 & Palisota hirsuta (Thunb.) K. Schum. & 40 & 8 & 20 & 2.02 & 1.63 & 1.81 & 5.46 & 0.03 & 0.02 & 0.50 \\
\hline 18 & Palisota ambigua (P. Beauv.) C.B. Cl. & 40 & 8 & 20 & 2.02 & 1.63 & 1.81 & 5.46 & 0.03 & 0.02 & 0.50 \\
\hline 19 & Costus lucanusianus J. Braun \& K. Schum. & 40 & 6 & 15 & 2.02 & 1.22 & 1.36 & 4.60 & 0.03 & 0.02 & 0.38 \\
\hline 20 & Anthocleista nobilis G. Don & 20 & 1 & 5 & 1.01 & 0.20 & 0.45 & 1.66 & 0.02 & 0.01 & 0.25 \\
\hline 21 & Psidium guajava Linn & 60 & 2 & 3.3 & 3.03 & 0.41 & 0.30 & 3.74 & 0.02 & 0.01 & 0.06 \\
\hline 22 & Selaginella myosurus (SW.) Alston. & 80 & 12 & 15 & 4.04 & 2.45 & 1.36 & 7.85 & 0.04 & 0.02 & 0.19 \\
\hline 23 & Craterispermum cerrinanthum Hiern. & 40 & 2 & 5 & 2.02 & 0.41 & 0.45 & 2.88 & 0.02 & 0.01 & 0.13 \\
\hline 24 & Carpoloba leutea G.Don. & 20 & 1 & 5 & 1.01 & 0.20 & 0.45 & 1.66 & 0.02 & 0.01 & 0.25 \\
\hline 25 & Triumfetta cordifolia A.Rich. & 80 & 4 & 5 & 4.04 & 0.82 & 0.45 & 5.31 & 0.03 & 0.02 & 0.63 \\
\hline 26 & Ipomoea involucrata P. Beauv. & 20 & 2 & 10 & 1.01 & 0.41 & 0.90 & 2.32 & 0.02 & 0.01 & 0.50 \\
\hline 27 & Landolphia dulcis (R. Br) Pichon & 20 & 1 & 5 & 1.01 & 0.20 & 0.45 & 1.66 & 0.01 & 0.01 & 0.25 \\
\hline 28 & Marattia fraxinea & 60 & 12 & 20 & 3.03 & 2.45 & 1.81 & 7.29 & 0.04 & 0.02 & 0.33 \\
\hline 29 & Panicum maximum Jacq & 80 & 20 & 25 & 4.04 & 4.08 & 2.26 & 10.38 & 0.05 & 0.03 & 0.31 \\
\hline 30 & Sida rhombifolia Linn. & 80 & 10 & 12.5 & 4.04 & 2.04 & 1.13 & 7.21 & 0.04 & 0.02 & 0.16 \\
\hline 33 & Chromolaena odorata (Linn) RM King \& Robinson & 60 & 16 & 26.7 & 3.03 & 3.26 & 2.41 & 8.70 & 0.04 & 0.02 & 0.45 \\
\hline 34 & Aspilla africana (Pers) CD Adams. & 40 & 30 & 75 & 2.02 & 6.11 & 6.78 & 14.91 & 0.06 & 0.03 & 1.88 \\
\hline 35 & Heterotis rotundifolia (Sm.) Jac. Fel. & 100 & 20 & 20 & 5.05 & 4.08 & 1.81 & 10.94 & 0.05 & 0.03 & 0.20 \\
\hline 36 & Platostoma africanum P. Beauv. & 20 & 6 & 30 & 1.02 & 1.22 & 2.71 & 4.94 & 0.03 & 0.02 & 1.50 \\
\hline 37 & Spillanthes filicaulis (Schum \& Thonn.) CD Adams & 40 & 16 & 40 & 2.02 & 3.26 & 3.62 & 8.90 & 0.04 & 0.02 & 1.00 \\
\hline 38 & Mariscus longbreateatus Cherm. & 20 & 4 & 20 & 1.02 & 0.82 & 1.81 & 3.64 & 0.02 & 0.01 & 1.00 \\
\hline 39 & Mariscus flabelliformis Kunth. & 20 & 6 & 30 & 1.01 & 1.22 & 2.71 & 4.94 & 0.03 & 0.02 & 1.50 \\
\hline 40 & Asystasia gagentica (Linn.) T. Anders. & 20 & 4 & 20 & 1.01 & 0.82 & 1.81 & 3.64 & 0.02 & 0.01 & 1.00 \\
\hline 41 & Alternanthera sessilis (Linn.) DC. & 40 & 14 & 35 & 2.02 & 2.85 & 3.16 & 8.03 & 0.04 & 0.02 & 0.88 \\
\hline 42 & Anchormanes giganteus Engl. & 20 & 1.6 & 8 & 1.01 & 0.33 & 0.72 & 2.06 & 0.01 & 0.01 & 0.40 \\
\hline 43 & Mariscus alternifolius Vahl. & 60 & 14 & 23.3 & 3.03 & 2.85 & 2.11 & 7.99 & 0.04 & 0.02 & 0.39 \\
\hline 44 & Perotis indica (Linn.) O. Ktze & 40 & 32 & 80 & 2.02 & 6.25 & 7.23 & 15.77 & 0.06 & 0.04 & 2.00 \\
\hline 45 & Oplimenus baumanii (Retz) P. Beauv. & 60 & 36 & 60 & 3.03 & 7.34 & 5.42 & 15.79 & 0.06 & 0.04 & 1.00 \\
\hline 46 & Diodia sermentosa & 60 & 10 & 16.7 & 3.03 & 2.04 & 1.51 & 6.58 & 0.03 & 0.02 & 0.28 \\
\hline 47 & Triumfetta rhomboidea & 40 & 2 & 5 & 2.02 & 0.41 & 0.45 & 2.88 & 0.02 & 0.01 & 0.13 \\
\hline 48 & Vernonia cineria & 20 & 4 & 20 & 2.02 & 0.82 & 1.81 & 3.64 & 0.02 & 0.01 & 1.00 \\
\hline 49 & Mitracarpus scarba & 60 & 8 & 13.3 & 3.03 & 1.63 & 1.20 & 5.86 & 0.03 & 0.02 & 0.22 \\
\hline 50 & Spermaccoci verticillata & 40 & 4 & 10 & 2.02 & 0.82 & 0.90 & 3.74 & 0.02 & 0.01 & 0.25 \\
\hline 51 & Axonopus compressus & 80 & 40 & 50 & 4.04 & 8.15 & 4.52 & 16.71 & 0.07 & 0.04 & 0.63 \\
\hline 52 & Emilia sonchifolia & 20 & 8 & 40 & 2.02 & 2.45 & 3.62 & 6.26 & 0.03 & 0.02 & 2.00 \\
\hline 53 & Aframommum danielli & 40 & 6 & 15 & 2.02 & 0.41 & 1.36 & 4.60 & 0.03 & 0.02 & 0.38 \\
\hline 54 & Triumfetta eriophlebia & 40 & 12 & 30 & 2.02 & 2.45 & 2.71 & 7.18 & 0.04 & 0.02 & 0.75 \\
\hline 55 & Funtumia africana & 20 & 2 & 10 & 1.01 & 0.41 & 0.90 & 2.32 & 0.02 & 0.01 & 0.50 \\
\hline 56 & Sida acuta & 40 & 12 & 30 & 2.02 & 2.45 & 2.71 & 7.18 & 0.04 & 0.020 & 0.75 \\
\hline 57 & Digitaria horizontalis & 60 & 10 & 16.7 & 3.03 & 2.04 & 1.51 & 6.58 & 0.03 & 0.019 & 0.28 \\
\hline 58 & Newbouldia laevis & 20 & 0.4 & 2 & 2.02 & 0.08 & 0.18 & 1.27 & 0.01 & 0.006 & 0.10 \\
\hline 59 & Lagenaria guineensis & $\begin{array}{l}60 \\
1980\end{array}$ & $\begin{array}{l}6 \\
490.6\end{array}$ & $\begin{array}{l}10 \\
1106.4\end{array}$ & 3.03 & 1.22 & 0.90 & $\begin{array}{l}5.15 \\
328.31\end{array}$ & 0.03 & 0.016 & 1.67 \\
\hline
\end{tabular}




\section{Discussion}

Floristic composition entails the individual species that occur in a stand or region. The nature of forest communities largely depends on the ecological characteristics in sites, species diversity and habitat status of species [40]. The study has shown that the area is richly endowed with floras of various strata and categories (trees, shrubs, herbs, lianers, and climbers). The greater abundance of herbaceous vascular species among the families is an indication of a secondary vegetation structure heterogeneous in nature. The heterogeneity of the study site vegetation is being attributed to a number of retrogressive processes such as the influence of human activities, the regeneration and floristic succession of study site. This has resulted to changes in vegetation structure in terms of abundance and species biodiversity. This corroborates the assertion by Cubizolle et al. [41] who observed human activity as an important agent influencing plant species biodiversity. Biodiversity has recently emerged as an issue of both scientific and political concern primarily because of an increase in extinction rates caused by human activities [42]. Several very large experiments have addressed the relationship between biodiversity, measured as species richness, and ecosystem function [43, 44, 45, 46, 47 48].

Ecological succession due to prevailing human factor and local environmental conditions such as hydrological dynamics has contributed to variation in floral diversity of the project site in terms of the emergence of heterogeneity under stable and moderate environmental factor. In general, plant community ecologists are concerned with patterns of species response to environmental gradients $[49,50,51]$ and tend to adopt (if only implicitly) a continuum approach to vegetation with its assumption of continuous change in composition with position in the multi-dimensional environmental space [52]. The study site has shown differences in the ranks of species in terms of frequency, abundance, density, diversity, importance value index and distribution pattern in different transect. The decreased shift in vegetation composition in dry season may have also ensued from the loss of annual species that may not have been environmentally adapted to such a seasonal change due to decline in moisture content (dry habitat) of the site. However, other parameters like topography, soil, climate, aspect, altitude and geographical location do influence the vegetation diversity of forest.

Frequency is a measure of the uniformity of distribution of a species; thus a low frequency indicates that a species is either irregularly distributed or rare in a particular stand or forest. Frequent distribution of plant density, cover, biomass per unit area, and height, are used as a measures for expressing biological abundance and biological dominance of vegetation, have also been used to describe species composition and spatial patterns of vegetation in different plant communities [53]. High frequency indicated more frequent distribution of species at the TDU site due to optimum environmental conditions such as the moisture content from the prevailing rainfall during the wet season. Species dominant during the different seasons at the site were $M$. subulatus, $A$. macrophylla, A. obanenesis, $E$. guineensis, $H$. madagascariensis, S. myosurus, L. macrophyllum, M. esculenta, P. pinnata, T. cordifolia, M. fraxinea, P. maximum, A. bussei, S. rhombifolia, S. garkaena, N. pumicicola, N. bisserata and $H$. rotundifolia for wet season, whereas in dry season $H$. rotundifolia showed high dominance at the site. Their dominance in a particular season is seen due to the availability of optimum conditions for their growth. Similar observations in context with the present study were also reported by Kukshal et al. [54] and Shameem et al. [55]. Thus it becomes evident that during these seasons frequent occurrence of species is mainly due to the presence of enough moisture and micro-nutrients [56]. Difference in the species composition from site to site is mostly due to micro-environmental changes [57]

It is essential to consider the environmental (e.g. climatic) control over the niche dimensions of individual species and patterns of local species richness (alpha diversity) before attempting to develop general principles for diversity theory. Species diversity is one of the most important characteristics of a community; it is a mechanism which generates stability. The nature of plant community at a place is determined by other that grows and develops in such environment [58]. The herbaceous nature of the site could also be a reflection of its life form transition stage with low shrubby and tree status at the time of sampling following human and environmental influences and conditions. This is in conformity with Shameen et al. [55] who observed high diversity of herb layer in the absence of much vegetation cover. Though similar decreases in species abundance and diversity have been recorded among species, this possibly could be due to anthropogenic gradient such as the local devegetation for fuel. However, the ecosystem still maintains the status of abundance, richness and evenness.

Species richness is defined as the number of species (i.e. trees, shrubs, vascular plants) per unit area. There is growing interest in quantifying patterns of species richness in tropical forest especially in forest fragments in a landscape mosaic. Landscape ecologists have used landscape metrics to estimate tree species richness with relatively little success because these variables do not take into account levels of natural and anthropogenic disturbance and the successional status of forests $[58,60,61]$. Numerous problems regarding the study of species richness need to be clarified, including the role of disturbance [62], and the relative importance of biotic versus abiotic factors [63, 64]. Huston [62] reviewed species richness extensively, and regarded patterns of species richness as being determined by the interaction of disturbance with environmental gradients and competitive exclusion. Although over any large region, distribution of species richness is likely to be governed by two or more environmental gradients [65, 66], species richness studies in relation to environmental gradients have been mainly single-factor studies.

This assertion could be reaffirmed by a macro-scale study, which has attributed species diversity richness to be a product 
of water energy dynamics [67]. Tropical studies have correlated the importance of moisture and related factors to species richness [68]. Similarly changes in the gradient of species richness pattern have also been commonly explained by factors such as climate productivity and other energy related factors [69]; while Lomolino [70] has pointed out that many components of climate and local environments such as temperature, precipitation, seasonality and disturbance regimes vary along species amplitudinal gradient which ultimately create variation in their richness. A re-analysis of data by Pausas and Austin [71] also suggests that environmental parameters (especially rainfall) may also be involved in determining species richness. This corroborates Currie [72] and O'Brien [73, 74]. Richerson \& Lum [75] found a positive logarithmic relationship between Californian plant-species richness and rainfall, the environmental variable that accounted for the greatest variance in species richness in their study. Knight et al. [76] and O'Brien [73] found positive correlation between mean annual rainfall and woody species richness in southern Africa. Gentry [77] found an increase in neotropical plant species richness with precipitation up to about $4000 \mathrm{~mm}$ where species richness reaches an asymeptote; however, he also noted that there were differences that could be explained by seasonality of rainfall and soil nutrients.

Spatial variation might be due to the soil type and its composition, elevation of sites, moisture contents of soil, nature of disturbance, human interference and isolation of study site populated regions [78]. Maximum density in wet season was presented by $M$. esculenta and A. compressus showed highest density in dry season. In general, density of herbaceous species varied at both site. These changes are attributed to changes in microclimate among other factors. Similar changes were also reported by llorkar and Kharti [79]. Studies conducted by Abdullah et al. [80] mentioned climatic factors as a reason to influence the distribution of species in certain habitats. Species richness and frequency of occurrence is further supported by the fact that landscapes are never static, their elements are in permanent temporal and spatial flux [81, 82].Comparatively, $O$. corymbosa and $A$. africana commonly showed maximum importance in wet season and $A$. compressus in dry season at the TDU site. However, this can be correlated with the assertion that the dominance of certain species during a particular period could be as the other co-dominant species do not reach maturity to complete their life cycle [55]. Also studies on analysis of vegetation have been carried out particularly in terms of phytosociology, species diversity, richness and abundance across different physiognomic unit in various parts of the world [83, 84-86, 87].This agrees with the postulation that the tropical rainforest is normally made up of intricate mixture of plants belonging to different plant families, genera and species.

The TDU site when compared based on hydrological seasonal variation, species similarity index was observed to show similar vegetation complex in terms of species composition with a maximum similarity of $56 \%$ despite the difference in species richness and abundance due to seasonal variation. During the wet season a high number of species occurred at the site, where as a declined trend was observed when the dry season approached resulting to dissimilarity $(67 \%)$ among the tree species community structure. This is related to differences in micro-climatic conditions and anthropogenic impacts influencing growth of different species at the site. A high degree of different dissimilarity among species community has also been reported by Verms et al. [88]. A considerable similarity was depicted in the herbaceous and shrubby community structure with an index similarity of $56 \%$ and $62 \%$ respectively.

\section{Potential and Associated Environmental Impacts and Mitigation Measures}

\subsection{Potential Impact}

The Thermal Desorption Unit (TDU) is imminent of environmental perturbation as it may affect the environmental segments of the surrounding. This is often attributed to emission of large particulate matters and dangerous gases which disperse over long distance causing ailment and disorder to plant and other living organisms including inhabitants of surrounding communities. However, the direct and indirect impact may result to:

1. Possible deposition of suspended particulate matter (SPM) on the flora composition of the project site and surrounding communities.

2. Because of the deposition on plant the photosynthetic process will be very badly affected resulting to chlorosis and other associated physiological stress.

3. This could also become a barrier to the transport and translocation of materials from the leaf to various parts of the plant species.

4. These particles may also penetrate inside the plants through leaves and branches thereby creating imbalance and localization of minerals, micro and major nutrients in the plants. All these affect the plant growth very badly.

5. Possible continuous and long term deposition of SPM may also cause the fertile and forest land to be unproductive for plants and farming around the surrounding communities.

6. The vegetation composition and structure may also be affected due to corrosive reactions of acid rain and precipitation.

7. The effect of a new activity on the area causing disturbance and interference in the host communities way of life.

8. Loss of habitat of many species.

9. Damages and general loss of biodiversity.

10. Thermal pollution could cause direct thermal shocks, changes in dissolved oxygen and redistribution of organisms in the local community.

11. Some of the plants can be killed bysudden temperature changes that are beyond the tolerance limit of their metabolic systems. 


\subsection{Mitigation Measures}

1. Fire outbreak in the area and surrounding vegetation shall be prevented.

2. Organizations shall institute a contingency plan (and should there be an existing one, need to be enforced effectively) which addresses the identification and protection of vulnerable and sensitive areas including the surrounding vegetation.

3. Vegetation clearing shall be minimized and limited to only required area (within the limit of the acquired land as this may influence negatively the existence of biodiversity of the area). Thus there is need for the creation and development of a buffer zone for flora that may have been displaced or lost their natural habitation as a result of such project.

4. Areas not directly used for the project should have the following mitigative measures viz.

- Vegetation will not be cut back to bare soil and should be left to decay naturally.

- Overhanging plant species will not be cut back

- There should be no chopping or removal of plant species of any kind and care should be taken to avoid damage to root zones in project operations.

- Branch removal may be necessary for the project site but this will be performed so as not to adversely affect the growth characteristics of the mangrove vegetation.

- Exploitation of forest resources by both contractors and staff of organization shall be prohibited. The management shall provide employment for the host communities by ensuring that majority of the unskilled and semi-skilled labour needed for the project execution shall be obtained from the surrounding communities.

- Routine inspection of project location and facilities shall be maintained to ensure facility integrity and guide against facility degeneration / collapse and pollutant release on vegetations of the area.

5. There is need for the management to institute an integrated conservation and development strategy considered as a valuable land use option to reconcile development and conservation objectives in rural areas for a profitable exploitation of resources by local residents.

The application of such a concept in communal lands outside protected areas has to meet two main requirements to ensure a sustainable implementation. First, it must rely on the support of local communities, through their active involvement in biodiversity management operations and hence decision making. Secondly, it requires precise and regular information on flora abundance and trends to ensure that management schemes are adaptive and allow for a sustainable use of biodiversity populations. Consequently, the implementation of a community-based conservation programme needs the development of a monitoring method that respects a compromise between its applicability by local communities, both in terms of finance and skills, and the reliability of results.

\section{Conclusion}

Understanding of vegetation structure and component of ecosystem is important in several environmental baseline and impact studies. Such investigation of vegetation is often carried out in order to establish the degree of imminent environmental hazard and recovery of such envisaged ecological hazard as could be the case with TDU Waste management project. In the event of uncontrolled and accidental discharge of SPM, a preconceived knowledge of the vegetation is helpful in the determination of long and short-term effects of such emission on all major ecological habitats of the affected area. It will also give adequate support and facilitate the identification and assessment of associated environmental problems such as socio-economic and health implications of ecological predicament that many arise as a result of the incident.

\section{References}

[1] Nwafor, J. C. Environmental Impact Assessment for sustainable development: The Nigerian perspective. EDPCA Publications Enugu, 2006.

[2] AUS AID. Baseline Study Guidelines. Australian AID Knowledge Warehouse, 2003.

[3] Collins, R. Ecological monitoring and biodiversity surveys at the Smithsonian Tropical Research Institute Boscas Deltoro Research Station. Caribean J. Sci. 41(3): 367 - 373. 2005.

[4] Minter, B. A, and Collins, J. P. Ecological ethics: building a new tool kit for ecologist and biodiversity managers. Conserve. Biol. 1803 - 1812. 2005.

[5] Edwin-Wosu, N. L. Eco-taxonomic Baseline Assessment of vegetation of Pirigbene -Obama - Agip oil field further development project in Southern Ijaw Local Government Area, Bayelsa State. Final Draft Report, NAOC / Sydney Gate-way (Nig) Limited. October, 2010.

[6] U. S. Environmental Protection Agency. Draft Guidance for Implementing Thermal Desorption Remedies at Superfund Sites. Memorandum from John J. Smith, Chief Design and Construction Management Branch. 1994a.

[7] U. S. Environmental Protection Agency. Vendor Information System for Innovative Treatment Technologies (VISITT Database), Version 4.0.Office of Solid Waste and Emergency Response, Washington, DC. 1994b.

[8] U. S. Environmental Protection Agency. Tech Trends: Thermal Desorption at Gas Plants. EPA-542-N-95-003.1995.

[9] U. S. Environmental Protection Agency. Technology Fact Sheet: A Citizen's Guide to Thermal Desorption. EPA 542-F-96-005.Technology Innovation Office. 1996.

[10] U. S. Environmental Protection Agency. Engineering Bulletin: Thermal Desorption Treatment. EPA/540/2-91/008.Superfund. 1991.

[11] Whitmore, T. C. An Introduction to Tropical Rainforest: Oxford University Press Inc., New York, 282p. 1998. 
[12] Myers, N. A.; Russell, C.; Mittermelert, G.; Mittermelert, A. B.; Gustavo, D.F. and Jennifer, K. Biodiversity hot spots for conservation priorities. Nature, 24: 853 - 858. 2000.

[13] El-Khouly, A. A. L. Effect of human activities on vegetation in Siwa Oasis. International Conference on Water Resources and Arid Environment, 112: 115 -143. 2004.

[14] Hopkin, B. Vegetation of the Olakemeji Forest Reserve, Nigeria J. Eco. 56:97-115. 1968.

[15] SAF. Forest Cover types of North America. Washington D. C. Society of American Foresters. 1954.

[16] Edwin-Wosu, N. L. Eco-taxonomic Post-impact Assessment of vegetation of GOI lake oil spillage (EIA SHEWING) Vegetation survey claim of Goi Community against Shell Petroleum Development Company (SPDC) in Gokana Local Govt. Area. 2011.

[17] Edwin-Wosu, N. L. Environmental Evaluation Study of Vegetation of the Disused Imo River, Nkpoku, Obigbo North, Ebubu Pipeline (ROW) in Rivers State / and Abia State, February, 2012. MACPHED / SPDC. Wet Season Draft Report. 2012a.

[18] Edwin-Wosu, N. L. EIA Baseline Study of Vegetation / Wildlife for the OBIAFU / OBRIKOM- Indorama Eleme Fertilizer Company Limited (IEFCL) Gas Delivery Pipeline Project, August 2012.Environmental \& Chemical Services Limited / IDORAMA ELEME PETROCHEMICAL COMPANY LIMITED. 2012b.

[19] Kinako, P. D. S. Ecology and conservation of natural resources. Belk publishers, Port Harcourt. Pp145. 1989.

[20] Burkill, H. M. Useful Plant of West Tropical Africa, Vol. 1 (2ed.). Royal Botanic Garden, Kew, London. 1985.

[21] Burkill, H. M. Useful Plant of West Tropical Africa, Vol. 2 (2ed.). Royal Botanic Garden, Kew, London. 1994.

[22] Burkill, H. M. Useful Plant of West Tropical Africa, Vol. 3 (2ed.). Royal Botanic Garden, Kew, London. 1995.

[23] Burkill, H. M. Useful Plant of West Tropical Africa, Vol. 4 (2ed.). Royal Botanic Garden, Kew, London. 1997.

[24] Burkill, H. M. Useful Plant of West Tropical Africa, Vol. 5 (2ed.). Royal Botanic Garden, Kew, London. 2000.

[25] Hutchinson, J.; Dalziel, J. M. Flora of West Tropical Africa, Vol. 1. (Part 1.) Crown Agents for oversea government and administrations, Millbank London. 1954.

[26] Hutchinson, J.; Dalziel, J. M. Flora of West Tropical Africa, Vol. 1. (Part 2.) Crown Agents for oversea government and administrations, Millbank London. 1958.

[27] Hutchinson, J.; Dalziel, J. M. Flora of West Tropical Africa, Vol. 2. Crown Agents for oversea government and administrations, Millbank London. 1963.

[28] Hutchinson, J.; Dalziel, J. M. Flora of West Tropical Africa, Vol. 3. (Part 1) Crown Agents for oversea government and administrations, Millbank London. 1968.

[29] Hutchinson, J.; Dalziel, J. M. Flora of West Tropical Africa, Vol. 3. (Part 2.) Crown Agents for oversea government and administrations, Millbank London. 1972.

[30] Keay, R. W. J. Trees of Nigeria. Clarendon Press. 1989.
[31] Austin, M. P. and Greg-smith, P. The application of quantitative method to vegetation survey J.Ecol. 56:851-884. 1968.

[32] Kershaw, K. A. Quantitative and Dynamic Plant Ecology. $2^{\text {nd }}$ Edition, Edward Arnold, London pp 305, 1975.

[33] Pryor, L. D. Australian endangered species. Eucalyptus Australian National Parks and Wildlife Services Special Publication (5) Canberra 139 pp. 1981.

[34] Shukla, S. R. and Chandel, S. P. Plant Ecology. $4^{\text {th }}$ Edn. S. Chandel and Co. Ramnagar, New Delhi - 110055.197pp. 1980.

[35] Bonham, C. D. Frequency: Measurement of Terrestrial vegetation, John Wiley and Sons Inc New York, NY pp 90-96. 1989.

[36] Shannon, C. E. and Wienner, W. The mathematical theory of communications. Urbana, Illinois: University of Illinois Press. 1949.

[37] Misra, R. Ecology Workbook. Oxford \& IBH Publishing Co. Pvt Ltd., New Delhi. 244 pp. 1968.

[38] Curtis, J. T. and Cottam, G. Plant Ecology Work Book: Laboratory field reference manual. Bugess Publishing Co., Minnesota. 193 pp. 1956.

[39] Sorensen, T. A. A method of establishing groups of equal amplitude in plant sociology based on similarity of species content. K. Danake Videnk Biol. Skr., 5: 1-34. 1948.

[40] Mohammed, A. and Al-Amin, M. Regeneration status in a proposed biodiversity conservation area of Bangladesh. Proceeding Pakistan Academy Science: 44(3): 165-172. 2007.

[41] Cubizolle, H. Tourman, A. Argant, J. Porteret, J. Oberlin, C. and Serieyssol, K. Organs of Europe biodiversity: palaeo-geographic signification of peat inception during the Holocene in the granite Eastern Massif Central (France). Landscape Ecology, 7: 211-227. 2003.

[42] Ehrlich, P. R. and Wilson, E. O. Biodiversity studies: science and policy. Science and policy. Science, 253: 758 - 762. 1991.

[43] Tilman, D. and Downing, J.A. Biodiversity and stability in grasslands. Nature, 367: 363 - 365. 1994.

[44] Naeem, S.; Thomson, L. J.; Lawler, S. P.; Lawton, J. H. and Woodfin, R. M. Declining biodiversity can alter the performance of ecosystem. Nature, 368: 734 - 737. 1994.

[45] Naeem, S.; Thomson, L. J.; Lawler, S. P.; Lawton, J. H. and Woodfin, R. M. Empirical evidence that declining species diversity may alter performance of terrestrial ecosystems. Proc. R. Soc. Land. B. 347: 249 - 262. 1995.

[46] Kareiva, P. Diversity begets productivity. Nature, 368: $686-$ 689. 1994.

[47] Kareiva, P. Diversity and sustainability on the prairie. Nature, 379: 673 - 674. 1996.

[48] Tilman, D. Biodiversity: population versus ecosystem stability. Ecology, 77: 350-363. 1996.

[49] Tilman, D.). Plant strategies and the structure and dynamics of plant communities. University Press, Princeton, NJ. 1988.

[50] Wisheu, I. C. and Keddy, P. A. Species richness-standing crop relationship along four lakeshore gradients: constraint on the general model. Canadian Journal of Botany, 67: 1609 - 1617. 1989. 
[51] Moore, D. R. J. and Keddy, P. A. The relationship between species richness and standing crop in wetlands: the importance of scale. Vegetation, 79: 99 - 106. 1989.

[52] Austin, M.P. The potential contribution of vegetation ecology to biodiversity research. Ecography, 22: 465 - 484.1999.

[53] Chen, J.; Shiyomi, M. and Yamamura, Y. Frequency distribution models for spatial patterns of vegetation abundance. Ecol. Mod., 211: 403 - 410. 2008.

[54] Kukshal, S.; Nautiyal, B. P.; Anthwal, A.; Sharma, A. and Bhat, A. B. Phytosociological investigation and lifeform pattern of grazing lands under pine canopy in temperate zone, Northwest, Himalaya, India. Res. J. Bot., 4: 55 - 69. 2009.

[55] Shameen, S. A.; Irfana, N. K. and Bhat, G. A. Comparative assessment of edaphic features and herbaceous diversity in lower Dachigham national park, Kashmir, Himalaya. Journal of Ecology and the Natural Environment, 3(6):196 - 204. 2011.

[56] Nanette, M. Deacon, L. and Robinson, C. Greater nitrogen and / or phosphorus availability increase plant species cover and diversity at a high arctic polar semi-desert. Polar Biol. 30: 559 $-570.2007$.

[57] Mishra, D.; Mishra, T. K. and Banerjee, S. K. Comparative phytosociological and soil physic-chemical aspects between managed and unmanaged lateritic land. Ann. For., 5(1): 16 25. 1997.

[58] Bliss, L. C. Rosine and lipid contents in alpine Tundra plants. Ecology, 43: 753 -757. 1962.

[59] Turner, I. M. and Corlett, R. T. The conservation value of small, isolated fragments of lowland tropical rain forest. Trends in Ecology and Evolution, 11: 330 - 333. 1996.

[60] Whitmore, T. C. Tropical rainforest disturbance, disappearance, and species loss. In Tropical Forest Remnants; W.F. Laurance and R. O. Bierregaard (Eds.) Chicago: University of Chicago Press, pp. 3 -12. 1997.

[61] Nepstad, D. C.; Versssimo, A.; Alencar, A.; Nobres, C.; Lima, E.; Lefebre, P.; Schlessinger, P.; Potter, C.; Moutinho, P.; Mendoza, E.; Cochrane, M. and Brooks, V. Large - scale impoverishment Amazonian forest by logging and fire. Nature, 398: 505 - 508. 1999.

[62] Huston, M. A. Biological diversity. The coexistence of species on changing landscapes. Cambridge University Press, Cambridge. 1994.

[63] Cornell, H. V. and Lawton, J. H. Species interactions, local and regional processes, and limits to the richness of ecological communities: a theoretical perspective. Journal of Animal Ecology, 61: 1-12. 1992.

[64] Austin, M. P. and Gaywood, M. Current problems of environmental gradients and species response curves in relation to continuum theory. Journal of vegetation science, 5: 473 482.1994 .

[65] Pausas, J. G. Species richness pattern in the understorey of Pyrenean. Pinus sylvestris forest. J. Veg. Sci. 5: $517-524$. 1994.

[66] Austin, M. P. Pausas, J. G. and Nicholis, A. O. Patterns of tree species richness in relaltion to environment in south-easthern New South Wales. Australian Journal of Ecology, 21: 154 164. 1996.
[67] O' Brien, E. M.; Whittaker, R. J. and Field, R. Climate and woody plant diversity in Southern Africa, relationships at species, genus and family levels. Ecography, 21, 495 - 509. 1988.

[68] Brown, J. H. Species diversity. Analytical biogeography (Eds.) A. A. Myers and P. S. Giller. Chapman and Hall, London. 1988.

[69] Grytness, J. A.; Birks, H. J. B. and Peglar, S. M. Plant species richness in Fennoscandia; evaluating relative importance of climate and history. Nordic Journal Botany 19, 489 - 503. 1999.

[70] Lomolino, M. V. Elevation gradient of species richness, historical and prospective views. Global Ecology and Biogeography, 10, 3 - 13. 2001.

[71] Pausas, J. G. and Austin, M. P. Patterns of plant species richness in relation to different environments: An appraisal. Journal of Vegetation Science, 12: 153 - 166. 2001.

[72] Currie, D. J. Energy and large scale patterns of animal and plant species richness. Am. Nat. 137: 27 - 49. 1991.

[73] O'Brien, E. M. Climatic gradient in woody plant species richness: towards an explanation based on an analysis of southern Africa's woody flora. J. Biogeogr. 20: 181 - 198. 1993.

[74] O' Brien, E. M. Water-energy dynamics, climate, and prediction of woody plant species richness: an interim general model. J. Biogeography, 25: 379 - 398. 1998.

[75] Richerson, P. J. and Lum, K. L. Pattern of species diversity in California: relations to weather and topography. Am. Nat. 116: $504-536.1980$.

[76] Knight, R. S.; Crowe, T. M. and Siegfried, W. R. Distribution and species richness of tree in southern African. J. S. Afr. Bot. 48: 455 - 480. 1982.

[77] Gentry, A. H. Changes in plant community diversity and floristics composition on environmental and geographical gradients. Ann. Mo. Bot. Gard. 75: 1 -34. 1988.

[78] Alhassan, A. B.; Chiroma, A. M. and Kundiri A. M. Properties and classification of soils of Kajimaram Oasis of Northeast Nigeria. Int. j. Agric. Biol., 8: 256 - 261. 2006.

[79] Ilorkar, V. M. and Khartri, P. K. Phytosociological study of Navegaon National Park, Maharashtra. Ind. For., 129(3):377 387. 2003.

[80] Abdullah, M. B.; Sanusi, S. S.; Abdul S. D. and Sawa, F. B. J. An assessment of the herbaceous species vegetation of Yankari Game Reserve, Bauchi, Nigeria. Am-Eur. J. Agric. Environ. Sci., 6(1): $20-25.2009$.

[81] Stemier, N. C. and Koliler, N. Effect of land scape pattern on species richness, a modeling approach. Agriculture, Ecosystem and Environment 93: 353 - 361. 2003.

[82] Brown, J. H. and Leband, D. N. Species imperilment and spatial patterns of development in the United States. Conservation Biology, 20(1): 239 - 244. 2006.

[83] Ganesh, T.; Ganesan, R.; Devy, M. S.; Davidar, R.; Bawa, K. S. Assessment of plant biodiversity at a mid-elevation evergreen forest of Kala Kad- Mundane Thura Tger Reserve, Western Ghats, India. Current Sciences 71: 379 - 392. 1996. 
[84] Pandey, S. K. and Shukla, R. P. Plant diversity and community patterns along the disturbance gradient in plantation forests of Sal (Shorearobusta Gaertn) Current Science 77: 814 - 818. 1999.

[85] Pandey, S. K. and Shukla, R. P. Plant diversity in managed Sal (Shorearobusta Gaertn) forest of Gorakhpur, India: species compositon, regeneration and conservation. Biodiversity and Conservation 12: 2295 - 2319. 2003.

[86] Pandey, S. K. and Shukla, R. P. Plant community and diversity patterns within the forested landscape of north-eatern U. P. Indian Forester 131: 1217 - 1226. 2005.
[87] Tripathi, S. L. and Shukla, R. P. Effect of clipping and grazing on the various vegetational parameters of grass-land communities of Gorakhpur. Tropical Ecology 48: 61 - 70. 2007.

[88] Verma, A. E.; Kapoor, K. S.; Rawat, R. S.; Subramani, S. P. and Kumar, S. Analysis of plant diversity in degraded and plantation forests in Kunihar forest division of Himachal Pradesh. Ind. J. For., 28 (10): 11 - 16. 2005. 